\title{
Uncertainties in direct neutron capture calculations due to nuclear structure models
}

\author{
T. Rauscher ${ }^{a *}$, K.-L. Kratz ${ }^{\mathrm{b}}$, H. Oberhummer ${ }^{\mathrm{c}}$, J. Dobaczewski ${ }^{\mathrm{d}}$, P. Möller ${ }^{\mathrm{e}}$, and M. \\ Sharma ${ }^{\mathrm{f}}$ \\ anstitut für Physik, Universität Basel, \\ Klingelbergstr. 82, CH-4056 Basel, Switzerland \\ bInstitut für Kernchemie, Universität Mainz, \\ Fritz-Strassmann-Weg 2, D-55099 Mainz, Germany \\ ${ }^{\mathrm{c}}$ Institut für Kernphysik, TU Wien, \\ Wiedner Hauptstr. 8-10, A-1040 Wien (Vienna), Austria \\ ${ }^{\mathrm{d}}$ Institute for Theoretical Physics, Warsaw University, \\ ul. Hoza 69, 00-681 Warsaw, Poland \\ eP. Moller Scientific Computing and Graphics Inc., \\ Los Alamos, New Mexico, USA \\ ${ }^{\mathrm{f}}$ Dept. of Physics, Kuwait University \\ Kuwait 13060
}

The prediction of cross sections for nuclei far off stability is crucial in the field of nuclear astrophysics. For spherical nuclei close to the dripline the statistical model (HauserFeshbach) approach is not applicable and direct contributions may dominate the cross sections. For neutron-rich, even-even Sn targets, we compare the resulting neutron capture cross sections when consistently taking the input for the direct capture calculations from three different microscopic models. The results underline the sensitivity of cross sections calculated in the direct model to nuclear structure models which can lead to high uncertainties when lacking experimental information.

\section{INTRODUCTION}

Explosive nuclear burning in astrophysical environments produces unstable nuclei which again can be targets for subsequent reactions. Most of these nuclei are not accessible in terrestrial labs or not fully explored by experiments, yet. For the majority of unstable nuclei the statistical model (Hauser-Feshbach) can be used to determine the cross sections. However, for nuclei close to the dripline the level density becomes too low to apply the statistical model [1] and contributions of a direct interaction mechanism (DI)

\footnotetext{
*APART fellow of the Austrian Academy of Sciences
} 


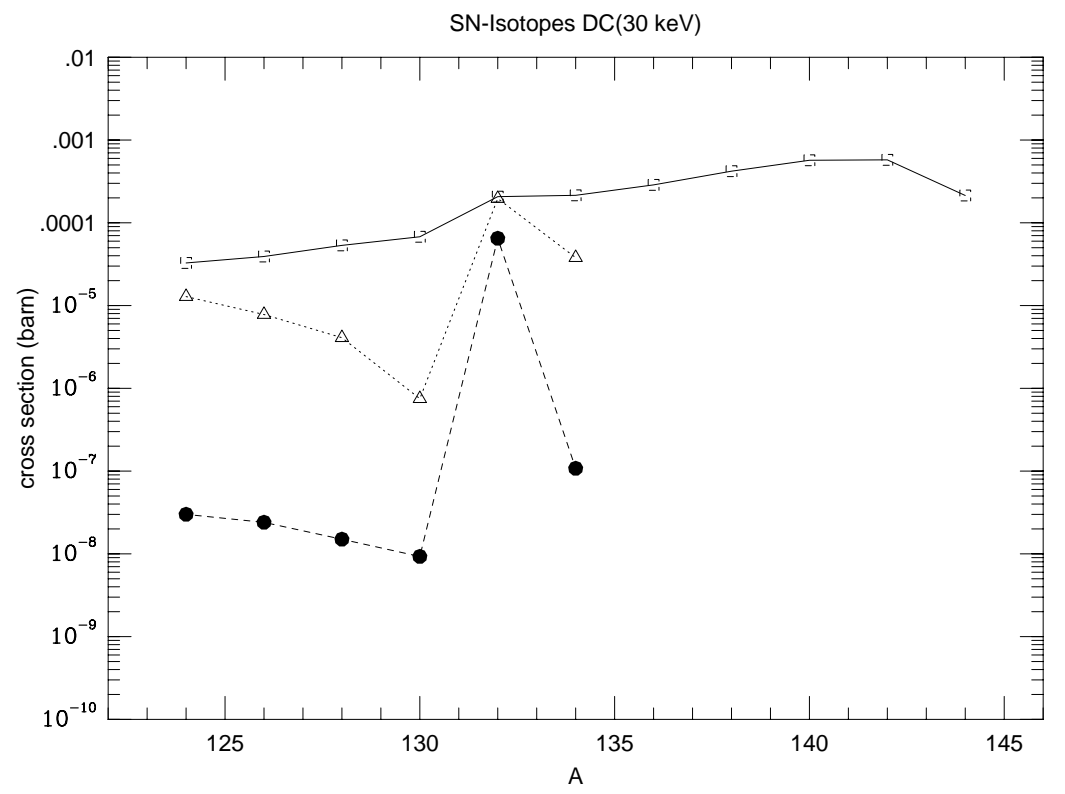

Figure 1. Direct-capture cross sections at $30 \mathrm{keV}$ for different $\mathrm{Sn}$ isotopes. Levels and masses are calculated with models by Sharma et al. [3] (triangles), Möller et al. [4] (dots), and Dobaczewski et al. [2] (squares). The lines are drawn to guide the eye.
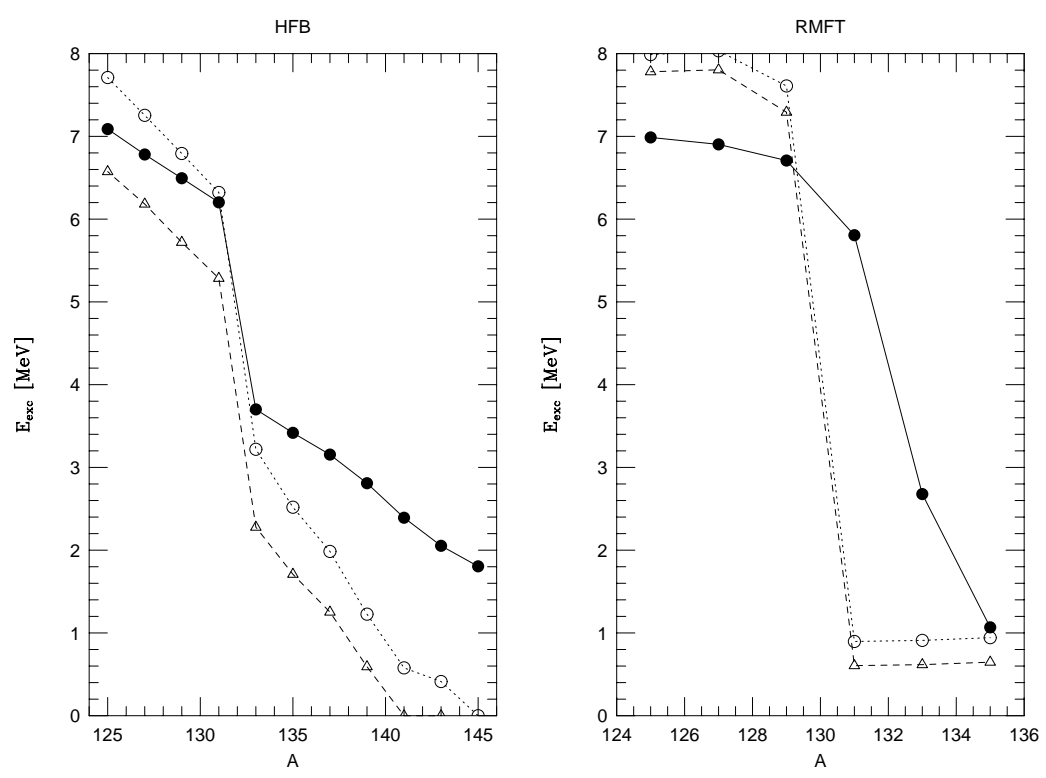

Figure 2. Dependence of level energies on mass number for the even-odd isotopes ${ }^{125-135} \mathrm{Sn}$ in the RMFT model [3] (right) and for the isotopes ${ }^{125-145} \mathrm{Sn}$ in the HFB model [2] (left). Shown are the $1 / 2^{-}$state (open circles), the $3 / 2^{-}$state (triangles) and the calculated neutron separation energy (full circles). The lines are drawn to guide the eye. Note the different range in mass numbers in the two plots. 
may dominate the cross sections. The DI requires the detailed knowledge of energy levels (excitation energies, spins, parities), contrary to the statistical model which averages over resonances. Lacking experimental data, this information has to be extracted from microscopic nuclear-structure models.

We compare the results for direct neutron capture (calculated in the optical model [6]) on the even-even isotopes ${ }^{124-145} \mathrm{Sn}$ with energy levels, masses, and nuclear density distributions taken from different nuclear-structure models. The utilized structure models were a Hartree-Fock-Bogoliubov model (HFB) with SkP force [2], a Relativistic Mean Field Theory (RMFT) with the parameter set NLSH [3] and a Shell Model based on folded-Yukawa wave functions (FYSM) [4].

A similar study has already been performed for neutron-rich $\mathrm{Pb}$ isotopes [5].

\section{METHOD}

The cross sections were calculated in the optical model for direct capture [6], utilizing optical potentials derived by the folding procedure [7]. In the folding approach the nuclear target density is folded with an energy- and density-dependent effective nucleon-nucleon interaction in order to obtain the potentials for the bound and scattering states. Only one open parameter $\lambda$ remains which accounts for the effects of antisymmetrization and is close to unity. The densities required for the determination of the folding potentials were consistently calculated from the wave functions of the respective nuclear-structure model. For the bound states the strength parameter $\lambda$ was fixed by the condition to reproduce the given binding energy of the captured neutron. The value of $\lambda$ for the scattering potential was adjusted to yield the same value of $425 \mathrm{MeV} \mathrm{fm}^{3}$ [8] for the volume integral as determined from the experimental scattering data on stable Sn isotopes [9, 10].

In order to be able to directly compare the different models, all nuclei were assumed to be spherical and the spectroscopic factors were set to 1 .

\section{RESULTS AND DISCUSSION}

The results of the calculations for projectiles at $E_{\text {c.m. }}=30 \mathrm{keV}$ are summarized in Fig. 11. For each model we calculated the capture cross section only up to the r-process path. The most extreme location of the path (farthest away from the line of stability) is determined by neutron separation energies $E_{\mathrm{n}} \approx 2 \mathrm{MeV}$ [1]. Depending on the microscopic model, the path will then be located at higher or lower mass numbers $A$. In the case of RMFT and FYSM it will go through $A \approx 132-134$, for HFB the path will be shifted to considerably higher mass numbers $A \approx 142-144$. (The neutron dripline is also shifted to higher masses in the latter model.)

Similar effects as seen in the behavior of the $\mathrm{Pb}$ cross sections [5] can also be found for the Sn cross sections. The cross section can vary by order of magnitudes when going from one isotope to the next and also differ vastly between the different microscopic models.

As the capture to low-spin states $(J=1 / 2,3 / 2)$ accounts for the largest contributions to the cross section, the results are very sensitive to the presence of bound low-spin states. Since the microscopic models not only yield different masses (i.e. neutron separation energies) but also exhibit different behaviors of the level energies with changing mass, "jumps" and "gaps" can be seen with some models (RMFT, FYSM), whereas others 
(HFB) result in a smoother behavior of the capture cross sections in an isotopic chain. This is illustrated in Fig. 2, which shows the neutron separation energy and the excitation energy of the $1 / 2^{-}$and $3 / 2^{-}$states in RMFT and HFB. As long as both states are unbound in the RMFT, the cross sections remain low and only jump to higher values when those states become bound at the shell closure. As at least the $3 / 2^{-}$level is always bound in HFB, the cross sections show a smoother behavior. The variation in the FYSM cross sections can be explained in a similar way.

\section{CONCLUSION}

With this work we have underlined that the calculation of purely theoretical direct capture cross sections far from stability still contains a large error, even when using most recent nuclear-structure models. In the previously discussed case of $\mathrm{Pb}$ isotopes [5], the r-process path contains nuclei in or at the border of a region expected to be deformed, leading to higher level densities and thus favoring the compound nucleus mechanism. This is not true for neutron-rich isotopes in the Sn region, especially around the neutron magic number $N=82$ where the level density becomes too low for the statistical model. Therefore the neutron capture cross sections have to be calculated using input from nuclear-structure models and will be subject to the quoted uncertainties, even when the different models yield similar values for other nuclear properties, such as masses.

Similar problems may be encountered on the proton-rich side when predicting proton capture cross sections close to the proton dripline.

\section{REFERENCES}

1. T. Rauscher, F.-K. Thielemann and K.-L. Kratz, Memorie della Società Astronomica Italiana, in press.

2. J. Dobaczewski et al., Phys. Rev. Lett. 72 (1994) 981.

3. M.M. Sharma, G.A. Lalazissis, and P. Ring, Phys. Lett. B 317 (1993) 9.

4. P. Möller and J.R. Nix, Nucl. Phys. A361 (1981) 117.

5. T. Rauscher, R. Bieber, S. Lingner, and H. Oberhummer, AIP Conf. Proc. 327 (1995) 183.

6. K.H. Kim, M.H. Park and B.T. Kim, Phys. Rev. C 23 (1987) 363.

7. A.M. Kobos, B.A. Brown, R. Lindsay, and G.R. Satchler, Nucl. Phys. A425 (1984) 205.

8. W. Balogh, R. Bieber, H. Oberhummer, T. Rauscher, K.-L. Kratz, P. Mohr, G. Staudt, and M.M. Sharma, Proc. Europ. Workshop on Heavy Element Nucleosynthesis, E. Somorjai and Z. Fülöp (eds.), Inst. Nucl. Res. Hung. Acad. Sci., Debrecen 1994, p. 67.

9. S.F. Mughabghab, M. Divadeenam and N.E. Holden, Neutron Capture Cross Sections, Academic Press, New York, 1981.

10. Data base CINDA, NNDC, Brookhaven National Laboratory, Upton, USA.

11. F.-K. Thielemann, K.-L. Kratz, B. Pfeiffer, T. Rauscher, L. van Wormer, and M. Wiescher, Nucl. Phys. A570 (1995) 329c. 